Regions where $A P O E \varepsilon 4$ positive $>A P O E \varepsilon 4$ negative
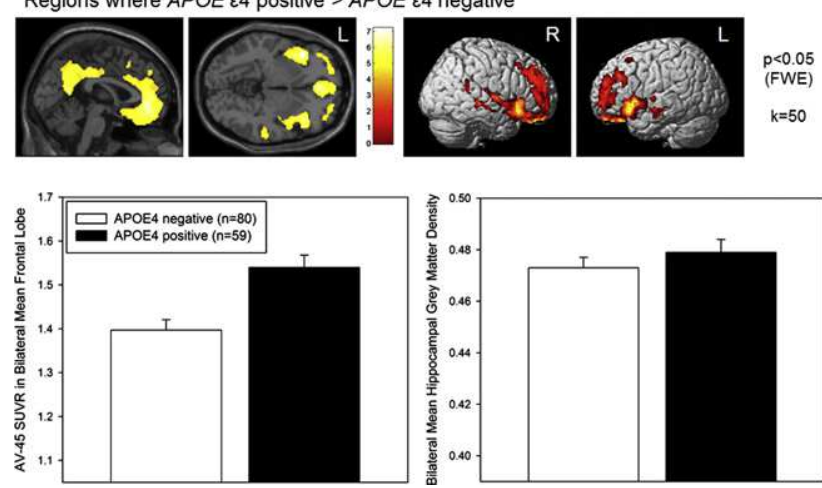

Figure 1. (A) Effect of $A P O E$ Genotype on Amyloid Deposition. (B) Frontal Amyloid by $A P O E$ Genotype. (C) Hippocampal Atrophy by $A P O E$ Genotype.

(E-MCI) provides an opportunity to evaluate the role of APOE E4 genotype on psychometric performance, amyloid deposition and neurodegeneration in patients with very mild clinical impairments. Methods: Baseline pre-processed AV-45 PET scans [2], 3T MRI scans [3], APOE genotype [4] and other quantitative phenotypes were downloaded from the ADNI website. Only participants categorized as EMCI $(n=139)$ at baseline from ADNI-GO/2 were included. MRI scans were processed using VBM, as previously described [5]. AV-45 scans were co-registered to the concurrent MRI and normalized to MNI space using parameters generated from MRI segmentation. Differences between APOE E4 allele positive and negative participants in AV-45 standardized uptake on a voxel-wise basis were assessed using a twosample t-test using SPM8. Region of interest (ROI) data was extracted from MRI and AV-45 scans using MarsBaR. Neuropsychological performance, cognitive complaints, and ROI data were compared between E4 positive and negative participants using independent-samples t-tests (SPSS 19.0). Results: Approximately $42 \%$ of the E-MCI participants (n $=139)$ were APOE E4 positive $(\mathrm{n}=59 ; 2$ E2E4, 48 E3E4, 9 E4E4; Table 1). The E4 positive E-MCI group showed lower performance on memory and global cognitive measures, but a trend toward fewer cognitive complaints, than APOE E4 negative participants $(n=80 ; 9$ E2E3, 71 E3E3). On AV-45 PET, E4 was associated with increased amyloid deposition, particularly in frontal and medial parietal lobar regions (Figure 1A; $P<0.05 \mathrm{FWE}, \mathrm{k}=50$ ). Similarly, ROI analyses indicated greater amyloid deposition in the frontal lobes of E4 positive E-MCI participants (Figure 1B; $P<0.001$ ). By contrast, the presence of E4 was not associated with greater hippocampal atrophy in this initial sample of E-MCI participants (Figure 1C). Conclusions: In E-MCI, APOE E4 genotypes are associated with greater amyloid deposition and cognitive changes, but not hippocampal neurodegeneration. References: [1] Corder (1993). [2] Jagust (2010). [3] Jack (2010). [4] Saykin (2010). [5] Risacher (2009).

Table 1

Demographics and neuropsychological test performance (Mean (SE))

\begin{tabular}{|c|c|c|c|}
\hline & $\varepsilon 4$ negative & $\varepsilon 4$ positive & $P$-value \\
\hline $\mathrm{n}$ & 80 & 59 & \\
\hline Age (years) & $71.97(0.86)$ & $70.81(1.00)$ & $\mathrm{ns}$ \\
\hline Gender (M, F) & 39,41 & 37,22 & $\mathrm{~ns}$ \\
\hline Education (years) & $15.96(0.30)$ & $15.73(0.35)$ & $\mathrm{ns}$ \\
\hline Handedness ( $\mathrm{R}, \mathrm{L})$ & 71,9 & 55,4 & $\mathrm{~ns}$ \\
\hline CDR-SB ${ }^{\mathrm{a}}$ & $1.11(0.08)$ & $1.38(0.09)$ & 0.022 \\
\hline ADAS-Cog Total ${ }^{\mathrm{a}, \mathrm{b}}$ & $10.95(0.53)$ & $13.79(0.61)$ & 0.0007 \\
\hline RAVLT Total $^{\mathrm{a}}$ & $41.76(1.03)$ & $37.54(1.20)$ & 0.009 \\
\hline
\end{tabular}

Table 1

Demographics and neuropsychological test performance (Mean (SE)) (Continued)

\begin{tabular}{lrrl}
\hline & $\varepsilon 4$ negative & \multicolumn{1}{c}{$\varepsilon 4$ positive } & $P$-value \\
\hline RAVLT Delayed Recall $^{\mathrm{a}}$ & $6.97(0.42)$ & $5.23(0.49)$ & 0.008 \\
Trail-Making B-A $^{\mathrm{a}, \mathrm{c}}$ & $53.90(4.86)$ & $69.38(5.65)$ & 0.042 \\
ECog Patient Total & $20.41(1.08)$ & $17.88(1.25)$ & $\mathrm{ns}$ \\
ECog Informant Total & $16.03(1.11)$ & $14.80(1.29)$ & $\mathrm{ns}$ \\
ECog-Mem Patient Total & $5.95(0.23)$ & $5.83(0.27)$ & $\mathrm{ns}$ \\
ECog-Mem Informant Total & $4.94(0.28)$ & $4.78(0.33)$ & $\mathrm{ns}$ \\
ECog-Lang Patient Total & $5.23(0.30)$ & $4.46(0.35)$ & $\mathrm{ns}$ \\
ECog-Lang Informant Total & $3.36(0.33)$ & $3.27(0.38)$ & $\mathrm{ns}$ \\
ECog-VS Patient Total & $2.58(0.27)$ & $1.85(0.32)$ & $\mathrm{ns}$ \\
ECog-VS Informant Total & $1.95(0.24)$ & $1.39(0.28)$ & $\mathrm{ns}$ \\
ECog-Exec Patient Total & $6.66(0.48)$ & $5.75(0.55)$ & $\mathrm{n}$ \\
ECog-Exec Informant Total & $5.78(0.50)$ & $5.36(0.58)$ & $\mathrm{ns}$ \\
\hline
\end{tabular}

\footnotetext{
${ }^{\mathrm{a}}$ Adjusted for age, gender, education, and handedness

${ }^{\mathrm{b}}$ Missing data for $1 \varepsilon 4$ negative participant

${ }^{\mathrm{c}}$ Missing data for 3 participants ( $2 \varepsilon 4$ negative, $1 \varepsilon 4$ positive)
}

\section{IC-P-099 ELEVATED PIB PRECEDES DEMENTIA IN AUTOSOMAL DOMINANT ALZHEIMER'S DISEASE: PIB, FDG AND ATROPHY IN THE DIAN COHORT}

Tammie Benzinger ${ }^{1}$, Tyler Blazey ${ }^{2}$, Robert Koeppe ${ }^{3}$, Clifford Jack $^{4}$, Marcus Raichle ${ }^{5}$, Yi Su ${ }^{5}$, Abraham Snyder ${ }^{5}$, Daniel Marcus ${ }^{5}$,

John Ringman ${ }^{6}$, Paul Thompson ${ }^{7}$, Bernardino Ghetti ${ }^{8}$, Andrew Saykin ${ }^{8}$, Stephen Salloway ${ }^{9}$, Steve Correia ${ }^{9}$, Keith Johnson ${ }^{10}$, Reisa Sperling ${ }^{10}$, Peter Schofield ${ }^{11}$, Colin Masters ${ }^{12}$, Christopher Rowe ${ }^{13}$,

Victor Villemagne ${ }^{14}$, Christopher Rowe ${ }^{15}$, Nick Fox ${ }^{16}$, Adam Brickman ${ }^{17}$, Richard Mayeux ${ }^{17}$, Ralph Martins ${ }^{18}$, Chester Mathis ${ }^{19}$, William Klunk ${ }^{19}$, Michael Weiner ${ }^{20}$, Randall Bateman ${ }^{21}$, Anne Fagan ${ }^{21}$, Alison Goate ${ }^{5}$, Nigel Cairns ${ }^{5}$, Virginia Buckles ${ }^{5}$, Krista Moulder ${ }^{5}$, John Morris ${ }^{5}$,

${ }^{1}$ Washington University School of Medicine, St. Louis, Missouri, United States, ${ }^{2}$ Washington University, St. Louis, Missouri, United States; ${ }^{3}$ University of Michigan, Ann Arbor, Michigan, United States; ${ }^{4}$ Mayo Clinic, Rochester, Minnesota, United States; ${ }^{5}$ Washington University, St. Louis, Missouri, United States; ${ }^{6}$ University of California, Los Angeles, Los Angeles, California, United States; ${ }^{7}$ University of California Los Angeles, Los Angeles, California, United States; ${ }^{8}$ University of Indiana, Indianapolis, Indiana, United States; ${ }^{9}$ Butler University, Providence, Rhode Island, United States; ${ }^{10}$ Brigham and Women's Hospital, Boston, Massachusetts, United States, ${ }^{11}$ University of New South Wales, Randwick-Sydney, Australia; ${ }^{12}$ University of Melbourne, Melbourne, Victoria, Australia; ${ }^{13}$ University of Melbourne, Melbourne, Australia; ${ }^{14}$ Austin Health, Melbourne, Australia; ${ }^{15}$ University of Melbourne, Heidelberg, Melbourne, Australia; ${ }^{16}$ University College of London, London, United Kingdom; ${ }^{17}$ Columbia University, New York, New York, United States, ${ }^{18}$ Edith Cowan University, Perth, Australia; ${ }^{19}$ University of Pittsburgh, Pittsburgh, Pennsylvania, United States; ${ }^{20}$ University of California, San Francisco, San Francisco, California, United States; ${ }^{21}$ Washington University School of Medicine, St. Louis, Missouri, United States.

Background: DIAN (Dominantly Inherited Alzheimer's Network) is an international longitudinal study of autosomal dominant Alzheimer's Disease (ADAD). In addition to clinical, cognitive and psychometric testing, participants undergo serial multi-modal imaging. Methods: 120 participants representing a mix of non-carrier and carriers in both the presymptomatic and symptomatic stages of $\mathrm{AD}$ underwent PIB, FDG PET and MRI. Cohorts were determined based on genetic status, dementia severity (Clinical Dementia Rating, CDR), and estimated time to dementia onset (TDO, based on parental age of onset). All imaging exams were transformed and processed in a common atlas 


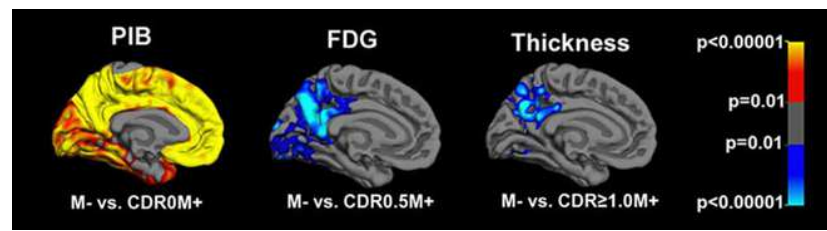

Table 1

\begin{tabular}{lllll}
\hline & $\begin{array}{l}\text { Non-carriers } \\
\text { (M-) CDR 0 }\end{array}$ & $\begin{array}{l}\text { Carriers } \\
(\mathrm{M}+) \text { CDR 0 }\end{array}$ & $\begin{array}{l}\text { Carriers }(\mathrm{M}+) \\
\text { CDR 0.5 }\end{array}$ & $\begin{array}{l}\text { Carriers }(\mathrm{M}+) \\
\mathrm{CDR}>=1\end{array}$ \\
\hline $\mathrm{n}$ & 43 & 44 & 18 & 15 \\
Age* $_{\text {Estimated time }}$ & $39.90(9.02)$ & $34.84(9.08)$ & $42.17(10.95)$ & $47.67(8.63)$ \\
$\quad$ to dementia* & $-5.48(12.33)$ & $-12.02(8.47)$ & $-1.72(8.75)$ & $+2.27(8.02)$ \\
Gender & $\mathrm{M}=43 \%$ & $\mathrm{M}=36 \%$ & $\mathrm{M}=56 \%$ & $\mathrm{M}=60 \%$ \\
Education* & $15.05(2.49)$ & $14.61(2.62)$ & $13.50(2.31)$ & $12.27(1.98)$ \\
\hline
\end{tabular}

* Mean (standard deviation) in years

space using a combination of in-house software and FreeSurfer. Regions of interest were applied to volumetric T1-weighted MRI, FDG and PIB data. For each modality and cohort, a linear regression analysis was used to determine the effects of TDO on a vertex-by-vertex basis. Results: Differences in PIB binding between carriers $(\mathrm{M}+)$ and non-carriers (M-) start to diverge many years prior to symptom onset (conversion to CDR 0.5). PIB retention in non-demented carriers were significantly different from the non-carrier cohort in the caudate, putamen and thalamus and in every cortical grey matter region (Figure 1). The first areas of significant amyloid deposition include the caudate, the occipital lobe, and the frontal lobe. Significant findings for grey matter volumes, cortical thickness, and FDG were limited to those carriers with dementia $(\mathrm{CDR}>=0.5)$ and did not reach significance in the pre-symptomatic population. Conclusions: DIAN represents the largest cohort of families with ADAD studied to date. Similar to findings in sporadic $\mathrm{AD}$, elevated PIB retention precedes detectable atrophy and metabolic changes by decades. Unlike sporadic AD, there is particular involvement of the caudate, and occipital lobe/visual cortex.Figure 1: Lateral surface projection of cluster corrected $\mathrm{p}$-values from linear regressions for PIB, FDG, and cortical thickness, when differences first appear. With PIB, non-demented carriers (CDROM+) demonstrate widespread amyloid deposition (left). With FDG, differences are identified in the carriers with very mild cognitive changes (CDR 0.5, M+, middle). Changes in cortical thickness are only identified in the cohort with mild dementia $(\mathrm{CDR}>=1, \mathrm{M}+$ ).

\section{IC-P-100 EFFECT OF THE APOE- $\varepsilon 4$ ALLELE ON LONGITUDINAL CHANGES IN CORTICAL THICKNESS IN NORMAL AGING}

Tara Madhyastha ${ }^{1}$, Paul Borghesani ${ }^{2}$, Elizabeth Aylward ${ }^{3}$, Monique Cherrier ${ }^{2}$, Mary Askren², Thomas Grabowski ${ }^{2}$,
K. Warner Schaie ${ }^{2}$, Sherry Willis ${ }^{2},{ }^{1}$ University of Washington, Seattle, Washington, United States; ${ }^{2}$ University of Washington, Seattle, Washington, United States; ${ }^{3}$ Seattle Children's Research Institute, Seattle, Washington, United States.

Background: Adults with at least one APOE E4 allele are at increased risk of earlier onset of memory decline and Alzheimer's Disease (AD). Neuroimaging cortical thickness biomarkers have been identified that are predictive of progression to AD. Most evidence for the APOE e4 allele modulation of cortical development is from cross-sectional data. This study examined the longitudinal effect of the APOE e4 allele on cortical thickness in normal aging. Methods: Data were obtained from participants in the Seattle Longitudinal Study (SLS), a cohort-sequential longitudinal study of the relationship between aging, health, cognition and lifestyle (Schaie, 2005). The sample included 111 participants, Mage $=67$ (age range $52-84$ ), imaged on 3 occasions over 4 years. In our sample, there were 32 APOE e4 carriers and 79 APOE e4 noncarriers. Magnetization prepared rapid gradient echo (MPRAGE) imaging was performed on a Philips 3.0 T Achieva scanner. Cortical reconstruction and volumetric segmentation was performed with the longitudinal pipeline of the FreeSurfer image analysis suite version 5.1.0 (http://surfer.nmr.mgh.harvard.edu/). Cortical thickness values for each of the 68 parcels defined by the Desikan parcellation (Desikan et al., 2006) were extracted by subject and timepoint. We fit a linear mixed effects model for each parcel that included fixed effects of intercept, age, and APOE e4 carrier status, and the interaction of age and APOE e4 carrier status to predict slope in cortical thickness of each parcel. Results: The mean estimated cortical thickness at age 60 (intercept) was thinner for APOE e4 carriers than APOE e4 non-carriers in the left inferior parietal parcel and left and right frontal pole. Slope differences (age X e4 carrier status interaction) were found in: left temporal pole and superior frontal regions, and right transverse temporal and caudal anterior cingulate (Figure 1). In all regions except the right transverse temporal, APOE e4 carriers had a steeper rate of decline than non-carriers. Conclusions: The APOE 4 allele modulates mean thickness and rates of change both in areas associated with normal aging and in areas associated with progression to $\mathrm{AD}$.
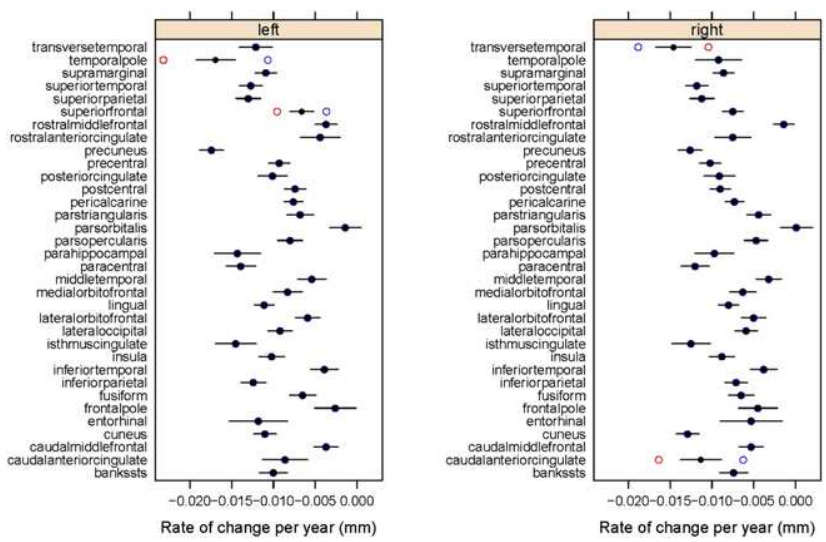